\title{
WIENER INTEGRALS OF MULTIPLE VARIATIONS
}

\section{MARGARET OWCHAR}

1. Introduction. $\mathrm{N}$. Wiener [1] has defined measure in the space $C$ of all real-valued functions $x(t)$ continuous on $0 \leqq t \leqq 1$ and vanishing at $t=0$. He defines a quasi-interval in the space as the set of all $x(t)$ in $C$ satisfying $a_{i}<x\left(t_{i}\right)<b_{i}, i=1,2, \cdots, n$, where $a_{i}$ and $b_{i}$ are real numbers such that $-\infty \leqq a_{i}<b_{i} \leqq+\infty, 0<t_{1}<t_{2}<\cdots<t_{n} \leqq 1$. Wiener then defines the measure of this quasi-interval to be

$$
\begin{array}{r}
\frac{1}{\left(\pi^{n} t_{1}\left(t_{2}-t_{1}\right) \cdots\left(t_{n}-t_{n-1}\right)\right)^{1 / 2}} \int_{a_{n}}^{b_{n}} \cdots \int_{a_{1}}^{b_{1}} \exp \left\{-\frac{u_{1}^{2}}{t_{1}}-\frac{\left(u_{2}-u_{1}\right)^{2}}{t_{2}-t_{1}}\right. \\
-\cdots-\frac{\left(u_{n}-u_{n-1}\right)^{2}}{t_{n}-t_{n-1}} d u_{1} \cdots d u_{n} .
\end{array}
$$

This definition leads in a familiar way to a Lebesgue type integral over C. R. H. Cameron and W. T. Martin have investigated the properties of this integral in various papers $[2 ; 3 ; 4 ; 5 ; 6]$, including its behavior under translation and more general linear and nonlinear transformations. They have defined a complete orthonormal set of functionals on $C$ which provides a means of development of nonlinear functionals in series, and have found methods of evaluating certain classes of Wiener in tegrals.

In this paper we consider the problem of integrating the $n$th variation of a functional $F(x)$ over the space $C$. The first variation, $\delta F(x \mid y)$, of the functional $F(x)$ with respect to $y(t) \in C$ is defined as $\delta F(x \mid y)$ $=d[F(x+h y)] /\left.d h\right|_{h=0}$, and the $n$th variation is defined as the first variation of the $(n-1)$ st variation; i.e.,

$$
\delta^{(n)} F\left(x \mid y_{1}, \cdots, y_{n}\right)=\left.\frac{d}{d h} \delta^{(n-1)} F\left(x+h y_{n} \mid y_{1}, \cdots, y_{n-1}\right)\right|_{h=0} .
$$

We also derive a result for the integration over $C$ of functionals related to the variations of $F(x)$, viz., functionals $F^{(n)}\left(x \mid t_{1}, \cdots, t_{n}\right)$ where $\int_{0}^{1} \cdots \int_{0}^{1} F^{(n)}\left(x \mid t_{1}, \cdots, t_{n}\right) y_{1}\left(t_{1}\right) \cdots y_{n}\left(t_{n}\right) d t_{1}$ $\cdots d t_{n}=\delta^{(n)} F\left(x \mid y_{1}, \cdots, y_{n}\right)$. It is known that if $\delta F(x \mid y)$ $=\int_{0}^{1} F^{(1)}(x \mid t) y(t) d t$, and $F^{(1)}(x \mid t)$ is continuous in $(x, t)$, where continuity in $x$ is defined in the uniform topology sense, then $F^{(1)}(x \mid t)$ is the Volterra derivative of $F(x)$ at the point $t, 0 \leqq t \leqq 1$.

Presented to the International Congress of Mathematicians, September 2, 1950; received by the editors April 23, 1951 and, in revised form, November 10, 1951. 
The problems we consider are a generalization of certain results proved by R. H. Cameron [2]. We shall have occasion to use one theorem from that paper several times; therefore we quote it here:

THEOREM 1a. Let $y_{0}(t) \in C$ be an absolutely continuous function with derivative $y_{0}^{\prime}(t)$ which is essentially of bounded variation ${ }^{1}$ on $0 \leqq t \leqq 1$, and let $F(x)$ be a Wiener summable functional over $C$ which has a first variation $\delta F\left(x \mid y_{0}\right)$ for all $x$ in $C$. Suppose also there exists an $\eta>0$ such that $\sup _{|h| \leq \eta}\left|\delta F\left(x+h y_{0} \mid y_{0}\right)\right|$ is Wiener summable in $x$ on $C$. Then it follows that both members of the following identity exist and are equal:

$$
\int_{c}^{w} \delta F\left(x \mid y_{0}\right) d_{w} x=2 \int_{c}^{w} F(x)\left[\int_{0}^{1} y_{0}^{\prime}(t) d x(t)\right] d_{w} x .
$$

It is understood that the symbol $\int_{c}^{w} F(x) d_{w} x$ represents the Wiener integral of the functional $F(x)$ over the space $C$.

2. Integral of the $n$th variation of a functional. We shall use the following notation in the succeeding theorems: $[n / 2]$ will denote the greatest integer less than or equal to $n / 2 ; K_{n, j}(\mu, \nu, \rho)$ will mean a combination of $j$ pairs $\left(\mu_{1}, \nu_{1}\right), \cdots,\left(\mu_{j}, \nu_{j}\right)$, chosen from the numbers $1, \cdots, n$, while $\rho_{1}, \rho_{2}, \cdots, \rho_{n-2 j}$ denote the remaining numbers; and $\sum_{K_{n, j}(\mu, v, \rho)}$ will mean the sum over the terms obtained by all possible choices of $K_{n, j}(\mu, \nu, \rho)$ disregarding order within the pairs as well as among the pairs and the remaining $n-2 j$ numbers. The number of terms in the sum is $n ! / 2^{i} j !(n-2 j) !$ for $0 \leqq j \leqq[n / 2]$.

THEOREM 1. Let $y_{1}(t), y_{2}(t), \cdots, y_{n}(t)$ be absolutely continuous functions in $C$, with derivatives $y_{1}^{\prime}(t), y_{2}^{\prime}(t), \cdots, y_{n}^{\prime}(t)$, which are essentially of bounded variation ${ }^{1}$ on $0 \leqq t \leqq 1$. Let $F(x)$ be a functional over $C$ such that

$$
F(x) \cdot \prod_{i=1}^{n}\left(1+\left|\int_{0}^{1} y_{i}^{\prime}(t) d x(t)\right|\right)
$$

is Wiener summable over $C$. Let the ith variation of $F(x)$ with respect to $y_{1}(t), \cdots, y_{i}(t), \delta^{(i)} F\left(x \mid y_{1}, y_{2}, \cdots, y_{i}\right)$, exist for $i=1,2, \cdots, n$, and let there exist $\eta_{i}>0$ such that

(1.2) $\sup _{|h| \leqq \eta_{i}}\left|\delta^{(i)} F\left(x+h y_{i} \mid y_{1}, \cdots, y_{i}\right)\right| \cdot \prod_{j=i+1}^{n}\left(1+\left|\int_{0}^{1} y_{j}^{\prime}(t) d x(t)\right|\right)$

${ }^{1}$ Here and elsewhere in this paper the requirement that a function be of "bounded variation" can be replaced by the requirement that it be "of class $L_{2}$ " if Stieltjes integrals are interpreted as Paley-Wiener-Zygmund integrals. 
is summable in $x$ on $C, i=1,2, \cdots, n$. (The product is understood to be unity when $i=n$.) Then it follows that both members of (1.3) exist and are equal:

$$
\begin{aligned}
& \int_{c}^{w} \delta^{(n)} F\left(x \mid y_{1}, \cdots, y_{n}\right) d_{w} x \\
&=\sum_{j=0}^{[n / 2]}(-1)^{j} 2^{n-j}\left(\sum_{K_{n, j}(\mu, v, \rho)} \prod_{k=1}^{j}\left[\int_{0}^{1} y_{\mu_{k}}^{\prime}(t) y_{p_{k}}^{\prime}(t) d t\right]\right. \\
&\left.\quad \int_{c}^{w} F(x) \prod_{i=1}^{n-2 j}\left[\int_{0}^{1} y_{\rho_{i}}^{\prime}(t) d x(t)\right] d_{w} x\right) .
\end{aligned}
$$

Proof. We shall prove this theorem by induction.

Assume that the theorem is true for $n$ and that $F(x)$ satisfies the conditions of the theorem for $n+1$. Then it is clear that $\delta F\left(x \mid y_{1}\right)$ satisfies the hypotheses of the theorem for $n$, and we conclude that

$$
\begin{aligned}
& \int_{c}^{w} \delta^{(n+1)} F\left(x \mid y_{1}, \cdots, y_{n+1}\right) d_{w} x \\
&=\sum_{j=0}^{[n / 2]}(-1)^{i} 2^{n-i}\left(\sum_{K_{n, j}(\mu, \nu, \rho)} \prod_{k=1}^{j}\left[\int_{0}^{1} y_{\mu_{k}}^{\prime}(t) y_{v_{k}}^{\prime}(t) d t\right]\right. \\
&\left.\cdot \int_{c}^{w} \delta F\left(x \mid y_{1}\right) \cdot \prod_{i=1}^{n-2 j}\left[\int_{0}^{1} y_{\rho_{i}}^{\prime}(t) d x(t)\right] d_{w} x\right),
\end{aligned}
$$

where $\mu_{k}, \nu_{k}$, and $\rho_{i}$ are chosen from the $n$ numbers $2,3, \cdots, n, n+1$. Now we have

$$
\begin{aligned}
\delta F\left(x \mid y_{1}\right) & \prod_{i=1}^{n-2 j}\left[\int_{0}^{1} y_{\rho_{i}}^{\prime}(t) d x(t)\right] \\
=\delta & {\left[F(x) \cdot \prod_{i=1}^{n-2 j}\left(\int_{0}^{1} y_{\rho_{i}}^{\prime}(t) d x(t)\right)\right] } \\
& \quad-F(x) \sum_{p=1}^{n-2 j}\left\{\int_{0}^{1} y_{\rho_{p}}^{\prime}(t) y_{1}^{\prime}(t) d t \prod_{i=1, i \neq p}^{n-2 j}\left(\int_{0}^{1} y_{\rho_{i}}^{\prime}(t) d x(t)\right)\right\}
\end{aligned}
$$

where the first term on the right represents the variation of the product with respect to $y_{1}(t)$, and the second term on the right is understood to vanish if $n=2 j$. After substitution of (1.5) in (1.4) both terms of the integrand of the Wiener integral on the right are summable since the second term is summable by (1.1), and the difference of the two terms is summable by (1.4). We can therefore write: 


$$
\begin{aligned}
\int_{c}^{w} \delta^{(n+1)} F\left(x \mid y_{1}, \cdots, y_{n+1}\right) d_{w} x \\
=\sum_{j=0}^{[n / 2]}(-1)^{i 2^{n-j}}\left(\sum_{K_{n}, j(\mu, \nu, \rho)} \prod_{k=1}^{j}\left[\int_{0}^{1} y_{\mu_{k}}^{\prime}(t) y_{\nu_{k}}^{\prime}(t) d t\right]\right. \\
\quad\left\{\int_{c}^{w} \delta\left[F(x) \cdot \prod_{i=1}^{n-2 j}\left[\int_{0}^{1} y_{\rho_{i}}^{\prime}(t) d x(t)\right]\right] d_{w} x\right) \\
\quad-\int_{c}^{w} F(x) \sum_{p=1}^{n-2 j}\left(\int_{0}^{1} y_{\rho_{p}}^{\prime}(t) y_{1}^{\prime}(t) d t\right. \\
\left.\left.\cdot \prod_{i=1, i \neq p}^{n-2 j}\left[\int_{0}^{1} y_{\rho_{i}}^{\prime}(t) d x(t)\right] d_{w} x\right\}\right) .
\end{aligned}
$$

Next we shall prove that $H(x) \equiv F(x) \prod_{i=1}^{n-2 j}\left[\int_{0}^{1} y_{\rho_{i}}^{\prime}(t) d x(t)\right]$ satisfies Theorem 1a. $H(x)$ is summable for $j=0,1, \cdots,[n / 2]$ by (1.1). Its first variation with respect to $y_{1}(t)$ exists as in (1.5), and it remains only to show that $\eta>0$ exists such that $\sup _{|h| \leqq \eta}\left|\delta H\left(x+h y_{1} \mid y_{1}\right)\right|$ is Wiener summable on $C$. To this end we write

$$
\begin{aligned}
\delta H\left(x+h y_{1} \mid y_{1}\right) & \\
= & \delta F\left(x+h y_{1} \mid y_{1}\right) \prod_{i=1}^{n-2 j}\left[\int_{0}^{1} y_{\rho_{i}}^{\prime}(t) d x(t)+h \int_{0}^{1} y_{\rho_{i}}^{\prime}(t) y_{1}^{\prime}(t) d t\right] \\
+ & F\left(x+h y_{1}\right) \sum_{p=1}^{n-2 j}\left\{\int_{0}^{1} y_{\rho_{p}}^{\prime}(t) y_{1}^{\prime}(t) d t\right. \\
\cdot \prod_{i=1, i \neq p}^{n-2 j} & {\left.\left[\int_{0}^{1} y_{\rho_{i}}^{\prime}(t) d x(t)+h \int_{0}^{1} y_{\rho_{i}}^{\prime}(t) y_{1}^{\prime}(t) d t\right]\right\} . }
\end{aligned}
$$

By (1.2) for $n+1$ we have that there exists $\eta_{1}>0$ such that the supremum for $|h| \leqq \eta_{1}$ of the first term on the right of (1.7) is summable, $j=0,1, \cdots,[n / 2]$. To show that the supremum for $|h| \leqq \eta_{2}$ of the second term on the right of (1.7) is summable in $x$ for some $\eta_{2}$ $<0$, we apply the mean value theorem to $F(x)$, obtaining $F\left(x+h y_{1}\right)=h \delta F\left(x+\theta h y_{1} \mid y_{1}\right)+F(x), 0<\theta<1$. Now, by (1.2) there exists $\eta_{3}>0$ such that

$$
\begin{aligned}
\sup _{|h| \leqq \eta_{3}} \mid h \delta F\left(x+\theta h y_{1} \mid y_{1}\right) \cdot \prod_{i=1, i \neq p}^{n-2 j}\left[\int_{0}^{1} y_{\rho_{i}}^{\prime}(t) d x(t)\right. & \\
& \left.+h \int_{0}^{1} y_{\rho_{i}}^{\prime}(t) y_{1}^{\prime}(t) d t\right] \mid
\end{aligned}
$$

is summable in $x$ on $C$, while by (1.1) likewise $\eta_{4}>0$ exists for which 


$$
\sup _{|h| \leqq \eta_{i}}\left|F(x) \prod_{i=1, i \neq p}^{n-2 j}\left[\int_{0}^{1} y_{\rho_{i}}^{\prime}(t) d x(t)+h \int_{0}^{1} y_{\rho_{i}}^{\prime}(t) y_{1}^{\prime}(t) d t\right]\right|
$$

is summable for $j=0,1, \cdots,[n / 2]$. Hence we may conclude that there exists $\eta_{2}>0$ such that the supremum of the second term on the right side of (1.7) for $|h| \leqq \eta_{2}$ is summable in $x$.

Thus we now apply the conclusion of Theorem 1 a to $H(x)$ in (1.6) and arrive at the following form:

$$
\begin{aligned}
& \int_{c}^{w} \delta^{(n+1)} F\left(x \mid y_{1}, \cdots, y_{n+1}\right) d_{w} x \\
& =\sum_{j=0}^{[n / 2]}(-1)^{j} 2^{n-j+1}\left(\sum_{K_{n, j}(\mu, v, \rho)} \prod_{k=1}^{j}\left[\int_{0}^{1} y_{\mu_{k}}^{\prime}(t) y_{v_{k}}^{\prime}(t) d t\right]\right. \\
& \left.\cdot \int_{c}^{w}\left\{F(x) \prod_{i=1}^{n-2 j}\left[\int_{0}^{1} y_{p_{i}}^{\prime}(t) d x(t)\right] \cdot \int_{0}^{1} y_{1}^{\prime}(t) d x(t)\right\} d_{w} x\right) \\
& -\sum_{j=0}^{[n / 2]}(-1)^{j} 2^{n-j}\left(\sum_{K_{n, j}(\mu, v, \rho)} \prod_{k=1}^{j}\left[\int_{0}^{1} y_{\mu_{k}}^{\prime}(t) y_{v_{k}}^{\prime}(t) d t\right]\right. \\
& \cdot \int_{c}^{w} F(x) \sum_{p=1}^{n-2 j}\left(\int_{0}^{1} y_{p_{p}}^{\prime}(t) y_{1}^{\prime}(t) d t\right.
\end{aligned}
$$

To combine these terms we let $j^{\prime}=j+1$ in the second term, remembering that when $2 j=2 j^{\prime}-2=n$, the summand is zero. We obtain (dropping primes):

$$
\begin{aligned}
& \int_{c}^{w} \delta^{(n+1)} F\left(x \mid y_{1}, \cdots, y_{n+1}\right) d_{w} x \\
&=\sum_{j=0}^{[n / 2]}(-1)^{j} 2^{n+1-i}\left(\sum_{K_{n}, j(\mu, \nu, \rho)} \prod_{k=1}^{j}\left[\int_{0}^{1} y_{\mu_{k}}^{\prime}(t) y_{\nu_{k}}^{\prime}(t) d t\right]\right. \\
&\left.\cdot \int_{c}^{w}\left\{F(x) \prod_{i=1}^{n-2 j}\left[\int_{0}^{1} y_{p_{i}}^{\prime}(t) d x(t)\right] \int_{0}^{1} y_{1}^{\prime}(t) d x(t)\right\} d_{w} x\right) \\
&+\sum_{j=1}^{[(n+1) / 2]}(-1)^{2^{2}} 2^{n+1-i}\left(\sum _ { K _ { n , j - 1 } ( \mu , \nu , \rho ) } \left\{\sum_{p=1}^{n+2-2 j} \int_{0}^{1} y_{\rho_{p}}^{\prime}(t) y_{1}^{\prime}(t) d t\right.\right. \\
&\left.\left.\cdot \prod_{k=1}^{j-1}\left[\int_{0}^{1} y_{\mu_{k}}^{\prime}(t) y_{v_{k}}^{\prime}(t) d t\right] \int_{c}^{w} F(x) \prod_{i=1, i \neq p}^{n+2-2 j}\left[\int_{0}^{1} y_{p_{i}}^{\prime}(t) d x(t)\right] d_{w} x\right\}\right)
\end{aligned}
$$

(where $K_{n, j}$ means $j$ pairs from $2, \cdots, n+1$ ). 
In the second term on the right-hand side the summation with respect to $j$ will go from 1 to $[(n+1) / 2]$ only, since if $n$ is odd, $[n / 2]+1=[(n+1) / 2]$, while if $n$ is even, the summand vanishes when $j=n / 2+1$. Combining the two terms we have the required result:

$$
\begin{aligned}
& \int_{c}^{w} \delta^{(n+1)} F\left(x \mid y_{1}, \cdots, y_{n+1}\right) d_{w} x \\
& =\sum_{j=0}^{[(n+1) / 2]}(-1)^{i 2^{n+1-j}}\left(\sum_{K_{n+1}, j(\mu, \nu, \rho)} \prod_{k=1}^{j}\left[\int_{0}^{1} y_{\mu_{k}}^{\prime}(t) y_{\nu_{k}}^{\prime}(t) d t\right]\right. \\
& \left.\int_{c}^{w} F(x) \prod_{i=1}^{n+1-2 j}\left[\int_{0}^{1} y_{p_{i}}^{\prime}(t) d x(t)\right] d_{w} x\right) \text {. }
\end{aligned}
$$

For,

$$
\begin{array}{r}
\sum_{K_{n, j-1}(\mu, v, \rho)}\left\{\sum_{p=1}^{n+2-2 j} \int_{0}^{1} y_{p_{p}}^{\prime}(t) y_{1}^{\prime}(t) d t \cdot \prod_{k=1}^{j-1}\left[\int_{0}^{1} y_{\mu_{k}}^{\prime}(t) y_{p_{k}}^{\prime}(t) d t\right]\right. \\
\left.\cdot \int_{c}^{w} F(x) \prod_{i=1, i \neq p}^{n+2-2 j}\left[\int_{0}^{1} y_{\rho_{i}}^{\prime}(t) d x(t)\right] d_{w} x\right\}
\end{array}
$$

is equal to the sum of

$$
\prod_{k=1}^{j}\left[\int_{0}^{1} y_{\mu_{k}}^{\prime}(t) y_{\nu_{k}}^{\prime}(t) d t\right] \cdot \int_{c}^{w} F(x) \prod_{i=1}^{n+1-2 j}\left[\int_{0}^{1} y_{\rho_{i}}^{\prime}(t) d x(t)\right] d_{w} x
$$

over all terms obtained by all the possible combinations of $j$ pairs $\left(\mu_{1}, \nu_{1}\right), \cdots,\left(\mu_{j}, \nu_{j}\right)$ chosen from the numbers $1,2, \cdots, n, n+1$ so that exactly one pair includes the number 1 , while $\sum_{K_{n, j}(\mu, v, \rho)}$ is the sum over all terms obtained by choosing $j$ pairs $\left(\mu_{1}, \nu_{1}\right), \cdots$, $\left(\mu_{j}, \nu_{j}\right)$ from the numbers $1,2, \cdots, n, n+1$ so that none of the pairs involves the number 1.

Now (1.8) is precisely the result of our theorem for $n+1$. This result has been verified for $n=1$ in Theorem 1a [2]. Hence the theorem is true for all values of $n$.

Note. It might be useful, from the standpoint of application, to note that the above theorem holds under the following set of conditions:

Let $F(x)$ be measurable and satisfy the inequality $|F(x)|$ $<A_{0} \exp \left(B_{0} \int_{0}^{1}[x(t)]^{2} d t\right), A_{0}$ any constant,$B_{0}<\pi^{2} / 4$. Let $y_{1}(t), \cdots, y_{n}(t)$ be defined as in Theorem 1, and let $\delta^{(i)} F\left(x \mid y_{1}, \cdots, y_{i}\right), i=1$, $2, \cdots, n$, be measurable and such that $\left|\delta^{(i)} F\left(x \mid y_{1}, \cdots, y_{i}\right)\right|<A_{i}$ $\cdot \exp \left(B_{i} \int_{0}^{1}[x(t)]^{2} d t\right), A_{i}$ any constant, $B_{i}<\pi^{2} / 4$. 
To show that these conditions imply the conditions of Theorem 1, we use the properties of exponentials and the Hölder inequality. The summability of the dominating functionals has been proved $[3 ; 5]$.

3. A special case of Theorem 1. Suppose $y_{1}(t), y_{2}(t), \cdots, y_{n}(t) \in C$ satisfy the hypotheses of Theorem 1 , and at the same time are such that the set of derivatives $y_{j}^{\prime}(t)$ is made up of $\nu$ subsets of identical elements-say $r_{i}$ derivatives equal to $y_{i}^{\prime}(t), i=1, \cdots, \nu$, and $\sum_{i=1}^{p} r_{i}=n$. (We can, if necessary, relabel the $y(t)$ to fit this notation.) Suppose, also, that the distinct members of the set of derivatives are orthonormal. Then Theorem 1 will hold with

$$
\begin{aligned}
\int_{c}^{w} \delta^{(n)} F\left(x \mid y_{1}, \cdots,\right. & \left.y_{n}\right) d_{w} x \\
& =\int_{c}^{w} F(x) \prod_{i=1}^{v}\left\{H_{r_{i}}\left(\int_{0}^{1} y_{i}^{\prime}(t) d x(t)\right)\right\} d_{w} x,
\end{aligned}
$$

where $H_{m}(u)$ is the Hermite polynomial defined as

$$
H_{m}(u)=(-1)^{m}\left\{\frac{d^{m}}{d u^{m}} e^{-u^{2}}\right\} e^{u^{2}},
$$

and expressible as

$$
H_{m}(u)=\sum_{j=0}^{[m / 2]}(-1)^{i} \frac{2^{m-2 i} m !}{j !(m-2 j) !} u^{m-2 i} .
$$

The proof of (1.9) is straightforward.

4. Integral of a functional related to the first variation. The theorem which follows will serve as a starting point for the induction proof of Theorem 3. It has been generalized from R. H. Cameron's Theorem III in [2] to the extent needed in another portion of the succeeding theorem.

THEOREM 2. Let $F(x)$ be a Wiener summable functional over $C$ such that

$$
F(x) \cdot \max _{0 \leqq t \leqq 1}|x(t)|
$$

is also Wiener summable. Let $F(x)$ have a first variation with respect to $y(t) \in C$, such that

$$
\delta F(x \mid y)=\int_{c}^{w} K(x \mid t) y(t) d t+\sum_{j=1}^{n} L_{j}(x) y\left(t_{j}\right)
$$


where $K(x \mid t)$ and $L_{j}(x)$ are measurable functionals over $C$ and $C \otimes[0,1]$ respectively, with the property that for each $y(t) \in C$ there exist $\eta=\eta(y)$ $>0$ for which

$$
\sup _{n \leqq \eta, 0 \leqq t \leqq 1}|K(x+h y \mid t)| \text { and } \sup _{h \leqq \eta, j=1,2, \cdots, n}\left|L_{j}(x+h y)\right|
$$

are summable in $x$ on $C$. Then it follows that $\int_{c}^{w} F(x) x(t) d_{w} x$ has an absolutely continuous derivative with respect to $t, 0 \leqq t \leqq 1$, except for jumps at $t_{j}, j=1,2, \cdots, n$, and this derivative vanishes at $t=1$ if $t_{j} \neq 1$, $j=1,2, \cdots, n$. Moreover,

$$
\int_{c}^{w} K(x \mid t) d_{w} x=-2 \frac{d^{2}}{d t^{2}} \int_{c}^{w} F(x) x(t) d_{w} x
$$

for almost all $t$ on $0 \leqq t \leqq 1$. In fact, (2.4) holds whenever its left member is continuous on $[0,1]$ except at $t_{1}, t_{2}, \cdots, t_{n}$.

The proof is closely analogous to that of Cameron's theorem.

\section{5 . Integral of a functional related to the $n$th variation.}

Theorem 3. Let $F(x)$ be a Wiener summable functional over $C$ such that

$$
F(x) \cdot \max _{0 \leqq t \leqq 1}|x(t)|^{n}
$$

is also Wiener summable over $C$.

Let the ith variation of $F(x)$ exist, $i=1,2, \cdots, n$, and let it be expressible as

$$
\begin{aligned}
\delta^{(i)} F\left(x \mid y_{1}, \cdots, y_{i}\right) \\
\quad=\int_{0}^{1} \cdots \int_{0}^{1} F^{(i)}\left(x \mid t_{1}, \cdots, t_{i}\right) y_{1}\left(t_{1}\right) \cdots y_{i}\left(t_{i}\right) d t_{1} \cdots d t_{i},
\end{aligned}
$$

where $F^{(i)}\left(x \mid t_{1}, \cdots, t_{i}\right)$ is continuous in $\left(x, t_{1}, \cdots, t_{i}\right)$, continuity in $x$ being in the uniform topology, and where $0 \leqq t_{j} \leqq 1, j=1,2, \cdots, i$. For each $y(t) \in C$ let there exist $\eta=\eta(y)>0$ for which

$$
\begin{aligned}
\sup _{|b| \leqq \eta, 0 \leqq t_{j} \leqq 1, j=1,2, \cdots, 8}\left|F^{(i)}\left(x+h y \mid t_{1}, \cdots, t_{i}\right)\right| \\
\\
\cdot\left(1+\max _{0 \leqq t \leqq 1}|x(t)|^{n-i}\right)
\end{aligned}
$$

is Wiener summable in $x$ on $C, i=1,2, \cdots, n$. Then it follows that 


$$
\begin{aligned}
\int_{c}^{w} F^{(n)}\left(x \mid t_{1}, \cdots, t_{n}\right) d_{w} x \\
=(-2)^{n} \frac{\partial^{2 n}}{\partial t_{n}^{2} \partial t_{n-1}^{2} \cdots \partial t_{1}^{2}} \int_{c}^{w} F(x) \prod_{i=1}^{n}\left\{x\left(t_{i}\right)\right\} d_{w} x
\end{aligned}
$$

for all $\left\{t_{i}\right\}$ on $[0,1], i=1,2, \cdots, n$, such that $t_{i} \neq t_{j}$ when $i \neq j$.

This theorem and Theorem 2 could be proved under the weaker condition that (3.3) and (2.3) be summable for a set of $y(t) \in C$ whose derivatives $y^{\prime}(t)$ are absolutely continuous on $[0,1]$ and vanish at $t=1$, and whose second derivatives $y^{\prime \prime}(t)$ are closed in $L_{2}(0,1)$.

Proof. This theorem, too, we shall prove by induction. Let us assume that the result holds for $n$. Then, under the same hypotheses for $n+1$ as quoted for $n$ above, we shall demonstrate that the theorem is true for $n+1$.

If we define $G(x)=F^{(1)}\left(x \mid t_{1}\right)$, we have

$$
F^{(n+1)}\left(x \mid t_{1}, \cdots, t_{n+1}\right)=G^{(n)}\left(x \mid t_{2}, \cdots, t_{n+1}\right),
$$

so that under hypotheses for $n+1$ and for fixed $t_{1}, G(x)$ satisfies the hypotheses of the theorem for $n$. That is, $G(x)$ and $G(x)$ - $\max _{0 \leqq t \leqq 1}|x(t)|^{n}$ are summable in $x$ on $C$ as a consequence of (3.3) with $n$ replaced by $n+1, i=1$, and $h=0$. Also, since $G^{(i)}\left(x \mid t_{2}, \cdots, t_{i+1}\right)$ $=F^{(i+1)}\left(x \mid t_{1}, \cdots, t_{i+1}\right)$, it is clear that

$$
\begin{array}{r}
\sup _{|h| \leqq \eta, 0 \leqq t_{j} \leqq 1, j=2,3, \cdots, t+1}\left|G^{(i)}\left(x+h y \mid t_{2}, \cdots, t_{i+1}\right)\right| \\
\cdot\left(1+\max _{0 \leqq t \leqq 1}|x(t)|^{n-i}\right) \\
\leqq \sup _{|n| \leqq \eta, 0 \leqq t_{j} \leqq 1, j=1,2, \cdots, t+1}\left|F^{(i+1)}\left(x+h y \mid t_{1}, \cdots, t_{i+1}\right)\right| \\
\cdot\left(1+\max _{0 \leqq t \leqq 1}|x(t)|^{n+1-(i+1)}\right) ;
\end{array}
$$

hence (3.3) is satisfied by $G(x)$. We conclude therefore that

$$
\begin{aligned}
\int_{c}^{w} F^{(n+1)}(x \mid & \left.t_{1}, \cdots, t_{n+1}\right) d_{w} x \\
= & (-2)^{n} \frac{\partial^{2 n}}{\partial t_{n+1}^{2} \cdots \partial t_{2}^{2}} \int_{c}^{w} F^{(1)}\left(x \mid t_{1}\right) \prod_{i=2}^{n+1}\left\{x\left(t_{i}\right)\right\} d_{w} x
\end{aligned}
$$

for all $\left\{t_{i}\right\}$ on $[0,1], i=2,3, \cdots, n+1$, such that $t_{i} \neq t_{j}$ when $i \neq j$.

Next we consider $H(x)=F(x) \prod_{i=2}^{n+1}\left\{x\left(t_{i}\right)\right\}$, and show that $H(x)$ 
satisfies the hypotheses of Theorem 2. $H(x)$ is summable since $F(x) \cdot \max _{0 \leqq t \leqq 1}|x(t)|^{n}$ is summable when (3.1) holds for $n+1$. Also $H(x) \cdot \max _{0 \leqq t \geqq 1}|x(t)|$ is summable since $|H(x)| \cdot \max _{0 \leqq t \geqq 1}|x(t)|$ $\leqq|F(x)| \cdot \max _{0 \leqq t \leqq 1}|x(t)|^{n+1}$ which is given summable in (3.1) for $n+1$. Furthermore,

$$
\delta H\left(x \mid y_{1}\right)=\delta F\left(x \mid y_{1}\right) \prod_{i=2}^{n+1}\left\{x\left(t_{i}\right)\right\}+\sum_{k=2}^{n+1}\left[F(x) \cdot y_{1}\left(t_{k}\right) \cdot \prod_{i=2, i \neq k}^{n+1}\left\{x\left(t_{i}\right)\right\}\right],
$$

wherefore we have

$$
\begin{aligned}
\delta H\left(x \mid y_{1}\right)= & \int_{0}^{1} F^{(1)}\left(x \mid t_{1}\right) y_{1}\left(t_{1}\right) \prod_{i=2}^{n+1}\left\{x\left(t_{i}\right)\right\} d t_{1} \\
& +\sum_{k=2}^{n+1}\left[F(x)\left\{\prod_{i=2, i \neq k}^{n+1}\left[x\left(t_{i}\right)\right]\right\} y_{1}\left(t_{k}\right)\right] .
\end{aligned}
$$

Now

$$
\begin{aligned}
\sup _{|h| \leqq \eta, 0 \leqq t_{1} \leqq 1} & \left|F^{(1)}\left(x+h y_{1} \mid t_{1}\right) \cdot \prod_{i=2}^{n+1}\left\{x\left(t_{i}\right)+h y_{1}\left(t_{i}\right)\right\}\right| \\
& \leqq \sum_{k=0}^{n} C_{k} \sup _{|n| \leqq \eta, 0 \leqq t_{1} \leqq 1}\left|F^{(1)}\left(x+h y_{1} \mid t_{1}\right)\right| \cdot \max _{0 \leqq t \leqq 1}|x(t)|^{n-k},
\end{aligned}
$$

where $C_{k}={ }_{n} C_{k} \eta^{k} \max _{0 \leqq t \leqq 1}\left|y_{1}(t)\right|^{k}$; but the expression on the righthand side is summable by (3.3) for $n+1$, with $i=1$. Also,

$$
\sup _{|n| \leqq \eta}\left|F\left(x+h y_{1}\right) \cdot \prod_{i=2, i \neq k}^{n+1}\left\{x\left(t_{i}\right)+h y_{1}\left(t_{i}\right)\right\}\right|, \quad k=2, \cdots, n+1,
$$

is summable over $C$. For, by the mean value theorem, $F\left(x+h y_{1}\right)$ $=h \delta F\left(x+\theta h y_{1} \mid y_{1}\right)+F(x)$, where $0<\theta<1$. Now

$$
\begin{aligned}
& \sup _{|h| \leqq \eta}\left|h \delta F\left(x+\theta h y_{1} \mid y_{1}\right) \prod_{i=2, i \neq k}^{n+1}\left\{x\left(t_{i}\right)+h y_{1}\left(t_{i}\right)\right\}\right| \\
& \leqq \eta \sup _{|n| \leqq \eta, 0 \leqq t_{1} \leqq 1}\left|F^{(1)}\left(x+\theta h y_{1} \mid t_{1}\right)\right| \cdot \max _{0 \leqq t_{1} \leqq 1}\left|y_{1}\left(t_{1}\right)\right| \\
& \text { - } \max _{0 \leqq t \leqq 1}\left|x(t)+h y_{1}(t)\right|^{n-1} \\
& \leqq{ }_{n-1} C_{\eta} \eta^{n} \sup _{|n| \leqq \eta, 0 \leqq t_{1} \leqq 1}\left|F^{(1)}\left(x+\theta h y_{1} \mid t_{1}\right)\right| \cdot \max _{0 \leqq t \leqq 1}|x(t)|^{j} \\
& \text { - } \max _{0 \leqq t \leqq 1}\left|y_{1}(t)\right|^{n-i}
\end{aligned}
$$


which is summable by (3.3). Likewise

$$
\begin{aligned}
\sup _{|n| \leqq \eta} \mid F(x) & \cdot \prod_{i=2, i \neq k}^{n+1}\left\{x\left(t_{i}\right)+h y_{1}\left(t_{i}\right)\right\} \mid \\
& \leqq|F(x)| \sum_{j=0}^{n-1}{ }_{n-1} C_{j} \eta^{n-j-1} \cdot \max _{0 \leqq t \leqq 1}|x(t)|^{j} \max _{0 \leqq t \leqq 1}\left|y_{1}(t)\right|^{n-j-1}
\end{aligned}
$$

which is summable by (3.1).

We apply Theorem 2 to $H(x)$ with

$$
K\left(x \mid t_{1}\right)=F^{(1)}\left(x \mid t_{1}\right) \prod_{i=2}^{n+1}\left\{x\left(t_{i}\right)\right\} \text { and } L_{j}(x)=F(x) \cdot \prod_{i=2, i \neq k}^{n+1}\left\{x\left(t_{i}\right)\right\}
$$

as in (3.6), and obtain

$$
\int_{c}^{w} F^{(1)}\left(x \mid t_{1}\right) \prod_{i=2}^{n+1}\left\{x\left(t_{i}\right)\right\} d_{w} x=-2 \frac{\partial^{2}}{\partial t_{1}^{2}} \int_{c}^{w} F(x) \prod_{i=1}^{n+1}\left\{x\left(t_{i}\right)\right\} d_{w} x
$$

for all $t_{1}$ on $[0,1]$ except at the points $t_{1}=t_{i}, i=2, \cdots, n+1$.

Finally, substituting for

$$
\int_{c}^{w} F^{(1)}\left(x \mid t_{1}\right) \prod_{i=2}^{n+1}\left\{x\left(t_{i}\right)\right\} d_{w} x,
$$

from (3.7) in (3.5), we obtain the desired result. To complete the induction, we note that the theorem holds for $n=1$ by Theorem 2 .

6. Applications. Let $z_{1}(t), z_{2}(t), \cdots, z_{2 n}(t)$ be functions which are of bounded variation on $[0,1]$, then it follows easily from Theorem 1 that

$$
\begin{aligned}
\int_{c}^{w} \prod_{i=1}^{2 n}\left[\int_{0}^{1} z_{i}(t) d x(t)\right] & d_{w} x \\
& =\frac{1}{2^{n}} \sum_{K_{2 n, n}(\mu, \nu)} \prod_{k=1}^{n}\left[\int_{0}^{1} z_{\mu_{k}}(t) z_{\nu_{k}}(t) d t\right]
\end{aligned}
$$

where $K_{2 n, n}(\mu, \nu)$ represents a combination of $n$ pairs $\left(\mu_{1}, \nu_{1}\right)$, $\left(\mu_{2}, \nu_{2}\right), \cdots,\left(\mu_{n}, \nu_{n}\right)$ from the $2 n$ numbers $1,2, \cdots, 2 n$, and $\sum_{K_{2 n, n}(\mu, \nu)}$ is the sum over terms obtained by all possible different choices of the $n$ pairs from these numbers.

This is a generalization of the Paley-Wiener-Zygmund formula [7]. Wiener has proved this result for $n=1$ in paper [1]. The induction 
follows through easily using Theorem 1 with $F(x)=1$.

If it is further assumed that $z_{1}(t), \cdots, z_{n}(t)$ are orthonormal on $0 \leqq t \leqq 1$, we have immediately

$$
\int_{c}^{w} \prod_{i=1}^{n}\left[\int_{0}^{1} z_{i}(t) d x(t)\right]^{2} d_{w} x=\frac{1}{2^{n}}
$$

It is hoped that the results which have been established in this paper will prove useful in the manipulation and evaluation of other Wiener integrals. In particular, it is hoped that the special case of Theorem 1 will lead to stronger convergence theorems on the FourierHermite development of functionals.

\section{REFERENCES}

1. N. Wiener, Generalized harmonic analysis, Acta Math. vol. 55 (1930) pp. 117258, especially pp. 214-234.

2. R. H. Cameron, The first variation of an indefinite Wiener integral, Proceedings of the American Mathematical Society vol. 2 (1951) pp. 914-924.

3. R. H. Cameron and W. T. Martin, Evaluation of various Wiener integrals by use of certain Sturm-Liouville differential equations, Bull. Amer. Math. Soc. vol, 51 (1945).

4. - The orthogonal development of non-linear functionals in series of FourierHermite functionals, Ann. of Math. vol. 48 (1948).

5. - Transformations of Wiener integrals under translations, Ann. of Math. vol. 45 (1944).

6. - Transformations of Wiener integrals under a general class of linear transformations, Trans. Amer. Math. Soc. vol. 58 (1945).

7. R. E. A. C. Paley, N. Wiener, and A. Zygmund, Notes on random functions, Math. Zeit. vol. 37 (1933) pp. 647-668, especially p. 650.

UNIVERSITY OF MinNESOTA 